\title{
Talk2Lab: The Smart Lab of the Future
}

\author{
Nicola J Knight ${ }^{1}$, Samantha Kanza ${ }^{2}$, Don Cruickshank ${ }^{3}$, William S Brocklesby ${ }^{4}$, Jeremy G Frey ${ }^{5}$.
}

\begin{abstract}
In today's world of advancing technology, pervasive and ubiquitous systems have become more common, and have been adapted for many different areas of use. The Internet of Things (IoT) movement has increased in popularity, and there are now many affordable, customisable smart devices available for various purposes. These devices are commonly found in the home, but have yet to infiltrate the scientific laboratory in the same way. Some laboratories do use IoT devices to control and monitor equipment, but smart laboratories just don't exist in the same way as smart homes, until now. This paper introduces the use cases for a smart integrated laboratory, and describes a prototype system, Talk2Lab, that was implemented in an experimental laser facility. Talk2Lab uses a combination of sensors, raspberry Pis, camera feeds and multiple interaction methods (voice, text, visual dashboards) to facilitate laboratory communication. It was evaluated by the laboratory users for usability, and to identify potential areas of expansion. The work to date illustrates the type of systems that can be created using IoT devices. From the results of the user evaluation we have conceptualised a future vision for Talk2Lab: a fully interconnected laboratory of the future.
\end{abstract}

Index Terms-Smart Lab, IoT, Alexa, Lab of the Future

\section{INTRODUCTION}

$\mathbf{T}$ AKE a moment to think, "If we redesigned the scientific laboratory with any of the possible technology of the 21 st century", "what could it look like?" Typically in research laboratories, notes are handwritten, instruments are isolated, measurements are often not digital, and there is limited system interaction [1]. However, integrating today's technology across all levels of the laboratory could create a seamless environment, revolutionising the laboratory. Although smart technology is becoming commonplace in everyday life, with many homes boasting the use of IoT devices and Smart Assistants such as Amazon Alexa or Google Home; this is not reflected in the average scientific research laboratory. Advances in technology can often be slow to reach researchers in their work environment, and currently most research laboratories remain primitive compared to the provisions of home automation systems. There are countless use cases for these new technologies; scientists frequently need access to systems or data in the laboratory from external locations, or need to access information but are in the middle of an experiment, or risk contaminating their technology with hazardous substances. It

\section{Manuscript received May 14, 2020}

${ }^{1}$ N. Knight, ${ }^{2}$ S.Kanza, ${ }^{3}$ D.Cruickshank, ${ }^{4}$ W.S. Brocklesby and ${ }^{5}$ J.G. Frey are all with the Faculty of Engineering and Physical Sciences at the University of Southampton, Highfield Campus, University Road, Southampton, SO17 1BJ, United Kingdom. n.knightesoton.ac.uk, s.kanzalsoton.ac.uk, dgceecs.soton.ac.uk, wsb@orc.soton.ac.uk, j.g.frey@soton.ac.uk

Copyright (c) 2020 IEEE. Personal use of this material is permitted. However, permission to use this material for any other purposes must be obtained from the IEEE by sending a request to pubs-permissions@ieee.org. would be a huge undertaking to entirely redesign a laboratory to provide a fully connected experience. However, integrating aspects of these technologies into a laboratory is achievable and the first step towards creating a fully connected lab.

We developed the prototype system Talk2Lab to investigate how scientists interact with their research environment; increase the connectivity of the lab, bring data together from multiple sources to facilitate better interaction, and ultimately to bring the laboratory into the smart age. Talk2Lab integrates sensors and smart devices in a laser laboratory, and links them up with a voice assistant and dashboards to provide scientists hands free access to their equipment data; enabling them to 'talk to the lab'.

This paper reviews previous work in Smart Laboratories and the Internet of Things (IoT), and states the generic use cases of a Smart Laboratory System. Talk2Lab's implementation is detailed, describing the laboratory environment and equipment, and explaining the different components of the system, the technologies that were used, and how the use cases were implemented for the chosen environment. The Talk2Lab system is then evaluated, followed by initial conclusions and a discussion of potential future endeavours of Talk2Lab, both for this specific implementation, and future implementations.

\section{RELATED WORK}

The concept of a 'Smart Lab' where technology is used to aid in lab processes, with connected laboratory instruments and the application of pervasive computing goes back 15 years. Frey et al [2] proposed the idea of a fully automated laboratory where different instruments can 'talk to each other'. This idea was built upon by Frey and Robinson [3], and Frey and Wilson [4]; both trials used IBM's middle-ware software, which uses the MQ telemetry transport (MQTT) $)^{1}$ protocol. MQTT uses the publish-subscribe methodology [5] to link up sensors in different labs to monitor certain conditions remotely. This work formed the very early basis of Talk2Lab.

Many laboratories have implemented linked systems; however, this typically involved utilising Laboratory Information Management Systems (LIMS) and Electronic Lab Notebooks (ELNs) to adhere to stringent requirements and regulations by linking equipment and managing data [6]. Early versions of this work by Hughes et al [7] used experiment plans to pre-populate linked tablet interfaces to facilitate quicker experiment data entry for scientists by making maximum use of information such as COSHH (Health and Safety) material prepared prior to experiments. Lütjohann et al [8] also developed a prototype ELN to link to electronic devices used in experiment. Additionally, various commercial solu-

\footnotetext{
${ }^{1}$ MQTT.org
} 
tions have emerged recently, demonstrating an uptake of linked laboratories in both academia and industry [9].

The use of IoT specific equipment in laboratories is slowly increasing. Shumate et al [10] used an arduino micro controller to connect a precision balance to a local network in order to automate dispensing accuracy. Whilst Linlin et al [11] used a combination of IoT devices and smart technologies to create an intelligent greenhouse monitoring system with temperature and equipment control. Additionally, the role of voice is now being considered in Smart Laboratories [12]. But there is still resistance to using ELNs, or indeed any screen-based interfaces in the laboratory, owing to accessibility and usability issues, particularly whilst conducting experiments [13], [14]. It is unsurprising therefore that voice in the laboratory has been considered as an alternative to physical interfaces where scientists must remove their gloves and access data via screens.

Laboratory systems that utilise voice interaction and IoT devices have started to make an impact in the field. Recently Helix [15]-[17] announced the creation of a voice-activated virtual lab assistant using IoT devices. However, thus far this system appears only to recall data from a fixed database rather than allowing the user to interact with data generated in the lab. It gives users access to pre-populated chemical information and procedures, but does not give any real-time or lab-specific data. In 2017 Perkel [18] reported the TetraScience grid, which uses sensors to monitor valuable lab samples to ensure that they remain at the right temperature. This system does provide specific real-time laboratory data, however it can only be accessed via a web browser, so despite providing timely data, it wouldn't necessarily be simple to access within the lab as it faces the same usage barriers as using screen-based interfaces within the lab. Other work has been undertaken which utilises the MQTT / node-RED framework that is used in the Talk2Lab system. These include work by Poongothai et al [19] which utilises IoT devices with MQTT / nodeRED to provide information on energy efficiency in a test lab environment, and work by Austerjost et al [20] which reports on a technical implementation of a voice layer on top of MQTT / node-RED.

Talk2Lab looks to integrate several of these elements to improve interaction across the whole laboratory ecosystem. The integration of multiple technologies enables access to realtime specific lab data and allows us to harness the strengths of the various systems in the different situations of a real multi-user working laboratory, creating a useful, timely and accessible IoT driven laboratory.

\section{Use CASES OF A SMART LABORATORY System}

Discussions were held with subject matter experts to identify how Talk2Lab could enhance the laboratory working environment, and several pieces of functionality were proposed. As a result, seven different use cases were identified for Talk2Lab, which were written up as generic use cases that could serve for various laboratories.

These use cases are detailed below, and their specific implementation for the first version of Talk $2 \mathrm{Lab}$ is detailed in Section V. The use cases are presented using a simplified template adapted from Fox and McGuinness [21].
Use Case Name: Voice Interaction with the Laboratory System

Goal: Provide hands-free access to information about the laboratory.

Summary: Often when users are working in the laboratory they are unable to access a computer interface, either due to location, using both hands for equipment or because they are handling hazardous substances. Being able to access laboratory information using voice removes these barriers and enables the user to gain access to necessary information in these situations.

Preconditions: Information needs to be well structured and labelled. A voice activated system needs to be set up (e.g. Amazon Alexa) and linked to different aspects of the laboratory.

Use Case Name: Text Interaction with the Laboratory System

Goal: Provide textual access to information about the laboratory.

Summary: Text interaction is useful in situations where audible interaction is not desired, e.g. in quiet shared offices, in noisy areas that disrupt audio quality, or for users with hearing impairments.

Preconditions: Information needs to be well structured and labelled. A text-queriable system needs to be setup and linked to the laboratory.

Use Case Name: Enquire about Laboratory Conditions

Goal: Retrieve information about laboratory environmental conditions.

Summary: Different environmental conditions can significantly impact equipment operation, the quality of experiment results, and can also indicate the possibility of equipment failure. Allowing users to retrieve instant and historical data can help explain unusual experimental behaviour.

Preconditions: Appropriate environmental sensors need to be installed in the laboratory. Different specification values need to be set (e.g. normal and abnormal conditions). A mechanism to make this information available needs to be put into place, e.g. voice or visual information retrieval.

Use Case Name: Enquire about Laboratory Equipment

Goal: Retrieve information about the status of equipment in the laboratory, both inside the laboratory and remotely.

Summary: Instant retrieval of information about laboratory equipment means users can immediately identify any equipment issues that may affect their work. This information could also be used during the experimental setup to aid the user, for example allowing the user to check when a piece of equipment had reached a certain state and was ready to be used.

Preconditions: Sensors need to be attached to the required pieces of equipment. Different specification values need to be set (e.g. working and not working). A mechanism for making this information available needs to be put into place, e.g. voice or visual information retrieval.

Use Case Name: Receive Warnings from the Laboratory

Goal: Alert users of unexpected conditions or potential laboratory issues.

Summary: Alerts could notify users of hazardous issues in the laboratory, or issues which may affect experimental results. If a user can rectify the issue during an experiment they may avoid repetition of the experiment. Providing users with different levels of severity of these warnings would allow them to effectively respond to the issue.

Preconditions: Appropriate sensors need to be installed in the laboratory and attached to relevant pieces of equipment. Multi level specification values need to be identified for each potential hazard or issue, with appropriate levels of severity assigned to each. A mechanism for sending this alert needs to be put into place e.g. text message, email, slack.

Use Case Name: View Camera Feeds

Goal: Display different camera feeds of the laboratory.

Summary: Camera feeds could be used for different purposes in the laboratory (e.g. to visualise experimental results or aid with experimental setup). Often setup requires multiple people due to the positions of equipment adjustment points and output viewpoints; and can be time consuming when done alone. Camera feeds showing this output near the adjustment points would simplify and speed up this setup process.

Preconditions: Networked camera feeds need to be set up in appropriate places, with display interfaces for the camera feeds to be viewed on. 
Use Case Name: View Laboratory Dashboards

Goal: Display multiple sources of laboratory information at once.

Summary: Combining different information sources can give users immediate insight into the laboratory status. This can be useful to laboratory supervisors who want to monitor what is going on, or to users about to enter the laboratory to conduct some work. The display can provide information critical to health and safety as well as the status of equipment and environmental conditions.

Preconditions: Data sources need to be well structured and labelled. Display interfaces need to be installed for dashboards to be viewed on. Build in redundancy to ensure real-time safety information is reliable.

\section{TALK2LAB ENVIRONMENT \& EQUIPMENT}

An experimental laser facility at the University of Southampton, run by Brocklesby and Frey, was selected for developing Talk2Lab. The laboratory comprises five areas; the main room, containing a laser system with four experimental beam lines, the majority of sensors, equipment and a mezzanine write up area; 2 entry areas; the chiller room; and the gas cupboard; shown in Fig.1.

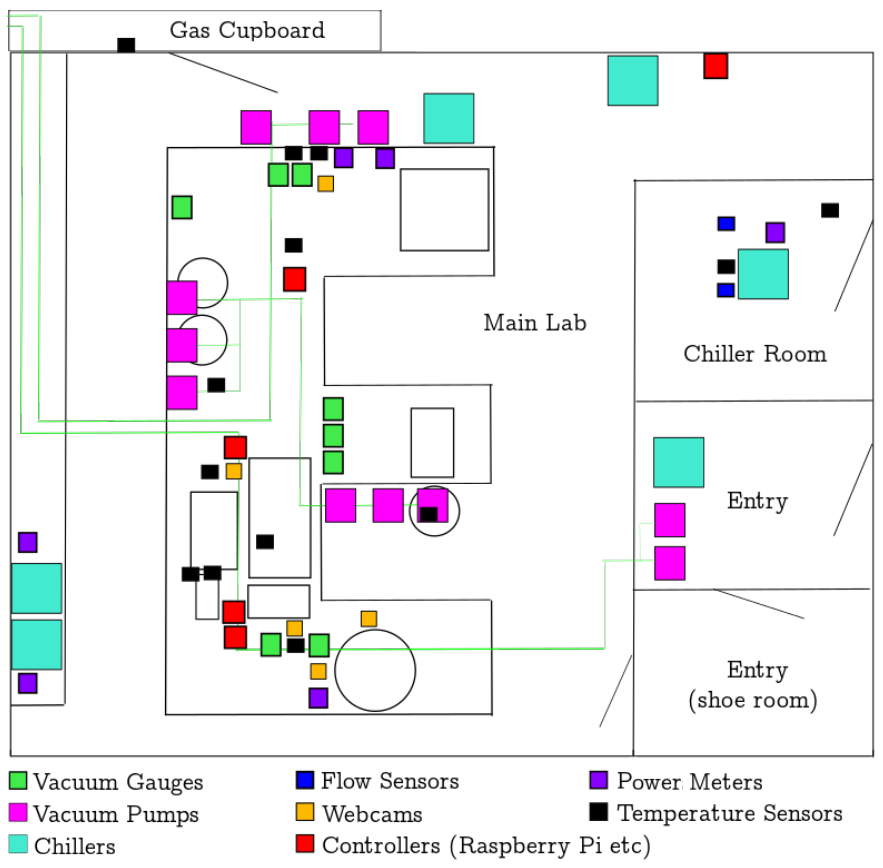

Fig. 1. Laser Facility Layout showing the different rooms and types of sensors

The class 4 laser setup is used in a variety of experiments using x-ray radiation for imaging and spectroscopy. The laser beam or indirect reflections of it can cause permanent eye damage and ignition of combustible materials, as such it requires a carefully controlled safety-conscious laboratory environment.

The laboratory equipment can be separated into three categories: safety-critical (maintains a safe working environment and minimises risk) e.g. the laser interlock that blocks the beam if the door is opened unexpectedly; experiment-specific e.g. imaging stages and vacuum chambers; and environmental (items monitoring lab conditions) e.g. temperature sensors.

\section{TALK2LAB IMPLEMENTATION}

Talk2Lab was designed as a real-time connected system, using a network of IoT devices layered with voice control, visualisation and text interaction to provide access and interaction with real-time laboratory data. It aims to enhance user-laboratory interaction with new methods that provide access to more data and improve laboratory monitoring, with interface mechanisms being designed so they don't interfere with users' work. When creating this system, a wide variety of technologies were available, ranging from open-source to purchased software to self-developed items. The technologies and hardware selected for the Talk2Lab system were chosen for their low cost, availability and ease of implementation.
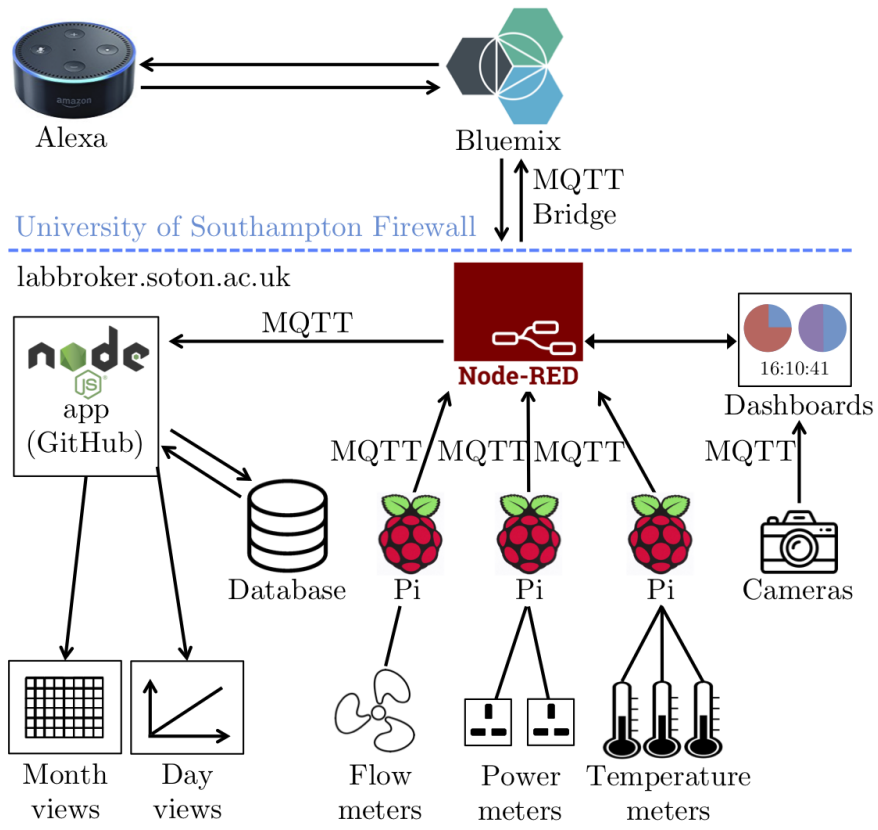

Fig. 2. Diagram illustrating the different technologies used in Talk2Lab

Fig. 2 illustrates the design of the Talk2Lab prototype. The primary communication within Talk2Lab is voice interaction utilizing the Amazon Echo Dot (Amazon's smart speaker linked to the voice activated personal assistant Alexa), and continuous display via dashboards. Text interaction was also developed via the Slack interface, however it did not have as flexible a communication method as via the Echo. The sensor system was implemented with a series of Raspberry Pis ${ }^{2}$ to collect and process readings from a various different sensors. These readings were relayed throughout the system via the lightweight messaging protocol MQTT [5], which works on a publish/subscribe basis, making it easy to add new feeds as required. The majority of the processing was carried out by our Node-RED ${ }^{3}$ hub to process voice interaction requests from the Echo Dot devices and text interaction via Slack [22]. Node-RED was also used to produce the dashboard displays.

Talk2Lab was developed such that it could be easily expanded and implemented in further locations. It uses readily

\footnotetext{
${ }^{2}$ https://www.raspberrypi.org/

${ }^{3}$ https://nodered.org/
} 
available commodity technologies where possible, that could be implemented with minimal customisation rather than bespoke systems. The next sections describe the Talk2Lab setup, how the different tools and technologies were implemented with respect to the use cases defined in Section III and detailing the additional help features that were also built.

\section{A. Talk2Lab Setup}

Prior to Talk2Lab, temperature sensors and power consumption monitors were installed in the laboratory to allow users to monitor environmental conditions. For Talk $2 \mathrm{Lab}$, additional sensors were installed to widen the scope of environmental monitoring with extra temperature readings (water and air), water flow readings, and extra power consumption. Network enabled cameras were installed, alongside two large display screens to provide visual output for both the camera feeds and dashboards. The cameras provided a mixture of readings, with some cameras providing readings following analysis of an image, and others positioned to remotely read values from equipment that may not otherwise be accessible. (The cameras encode their pictures as Base64 and send them via MQTT.)

Sensors were programmed to automatically obtain readings at set time intervals and publish them to a database (with Raspberry Pis and MQTT). This database holds all of the sensor data since the creation of Talk2lab, and was made accessible through a website created using the $\mathrm{d} 3 . \mathrm{js}{ }^{4}$ framework [23], which displays interactive graphs of the sensor data for selected time periods. These sensors were central to the first implementation of Talk2Lab as they provided underlying data, for scientists to retrieve real-time data about the lab.

Processing the growing set of sensor feeds highlighted the need for a defined structure of laboratory hardware, rather than the ambiguous naming schemes that often arise in university laboratories due to the cycle of users through them. In considering the structure, it was necessary to catalogue the hardware in the laboratory (including sensors, laboratory equipment and cameras). A hardware database was created, assigning each item a UUID (Universally unique identifier) to avoid duplication issues. Friendly names and nicknames were also added, with metadata including: location, device type, manufacturer, serial numbers and specifications for sensor alerts if available. Creating the item catalogue will allow automatic generation of interactions which pull values directly from the catalogue. Fig. 3 illustrates how laboratory equipment and their associated measurements are linked.

\section{B. Voice Interaction with Laboratory System}

A smart voice assistant was used to facilitate voice interaction, allowing users to interrogate the laboratory and retrieve real-time data in a hands-free manner. Amazon Alexa was selected for development purposes as custom interactions can easily be built within it. The Alexa system is installed on the Echo devices, processing voice requests and responses in the cloud. In order for the Echo device to interact with our processing system via Alexa Voice Services (AVS) a custom

${ }^{4}$ https://d3js.org/

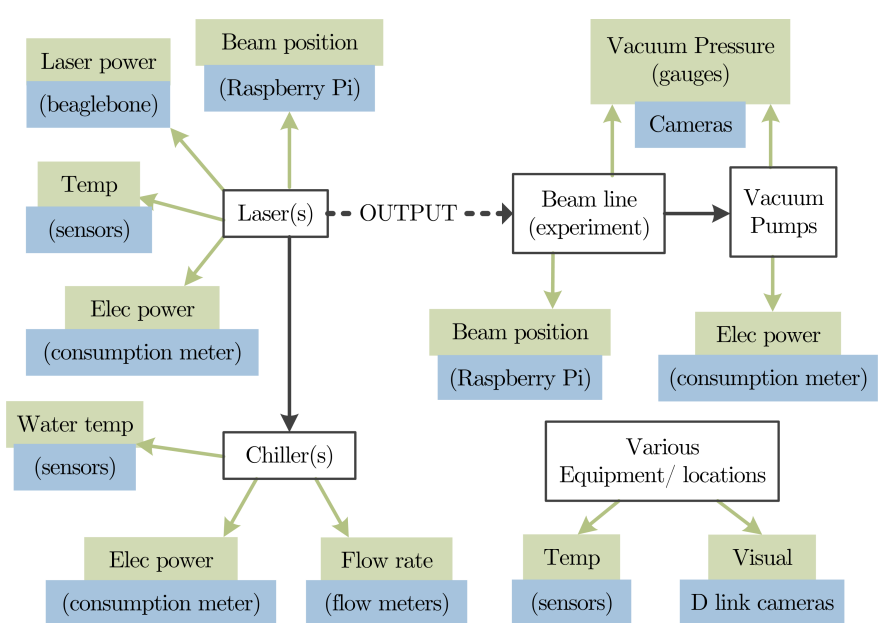

Fig. 3. Simplified map linking equipment and measurements in the lab. Black boxes - key items of equipment, green boxes - 'measurements', blue boxes devices taking measurement (also items of equipment)

Alexa skill was required. This skill defined the specifications for questions (intents) that would be asked, their required variables (slots) and the different options (slot values) that could be used. Multiple utterances can be defined within an intent, allowing for additional flexibility in speech patterns. Interaction through Alexa is triggered by spoken requests from a user. These take the form of request qualifier followed by a question in natural language. Fig. 4 shows examples of the Alexa request structure for our skill.

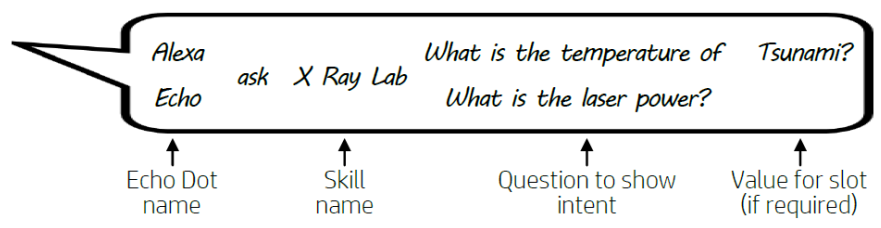

Fig. 4. Example structure of Alexa Requests

Alexa requests are pre-processed on the Amazon server, which uses speech-to-text and Natural Language Processing/Understanding (NLP/NLU) to try to identify the skill, intent and any slot values based upon the defined skill structure. Requests to our lab skill are then passed, in a structured form, to the Talk2Lab Node-RED server. This server consists of a set of nodes and node connections which create flows through the system, and it interfaces between the laboratory data and interaction systems. It pulls in data from multiple sources and allows information requests to be made through the Echo Dot.

The flow through Node-RED is initiated by an event e.g. an Alexa request or sensor reading. Each node in the flow then performs an operation such as retrieving or transforming data, or selecting an operation based on the data, before it passing it to the next node. The server has sections in the flow to process the Alexa request, retrieve the relevant data and produce the response. Given the incorporation of many intents for the different sensors and questions, the system requires several different pathways to retrieve the relevant data. A section of nodes in the system have also been set up to monitor sensor data. These subscribe to MQTT feeds published by sensors 
such as temperature, flow rate and power. When messages are received they pre-process (if necessary) and store the values in the system. They also monitor feeds for the laser beam position, power, wavelength and current user.

Using temperature as an example: A user asks 'Alexa, Ask $\mathrm{X}$ Ray lab, What is the temperature of Tsunami?' Alexa performs pre-processing; identifying the intent (GetTemperature) and the slot \& value (Tsunami) and passes the request to Talk2Lab. Node-RED directs the flow based on the GetTemperature request, and maps the slot value of Tsunami to the corresponding temperature sensor. The sensor's latest temperature reading is then retrieved, scaled and responded to with a timestamp to show if the data is up-to-date (e.g. 'The temperature of Tsunami is 19.3 degrees at 13:44'). This response is passed back to Alexa to be said to the user.

\section{Text Interaction with Laboratory System}

Voice interaction may not always be suitable e.g. in a quiet office space or particularly noisy environment. To combat this we also implemented a text interaction method via Slack to enquire about sensor readings. This utilised a Slack bot to monitor a Slack channel for posts (questions) and pass these to the Node-RED server for processing. Unlike Alexa requests, Slack messages are passed on in raw text form without any language processing; requiring identification and extraction of request parameters in Node-RED. If the requests use natural language, such as "What is the temperature of tsunami?", then NLP/NLU is required to identify the intent and parameters.

NLP/NLU engines exist that can be integrated with NodeRED, however most cloud engines (Amazon Alexa, IBM Watson) require paid subscriptions. An alternative is Open Source engines, but these often require more development and customisation. We circumvented the need for NLP by mandating a specific request structure of the format 'Intent:parameter1, parameter2 (if required)' e.g. 'Temperature:tsunami'; reducing processing complexity, and speeding up request entries. This enabled making retrieval requests for readings which mirrored the Alexa requests, however the strict input syntax was not a natural way of interacting. Spelling mistakes, or incorrect delimiters or spacing caused the system to generate errors. It would require significant expansion of error handling to cope with variations and potential syntax errors in user input to create a seamless Slack interface.

\section{Enquire about Laboratory Conditions}

Sensors were installed to monitor laboratory conditions including air/water temperatures, and water flow rates. These readings are useful to users when examining results or troubleshooting laboratory issues. A separate feed also monitors the current laboratory user, so other users know if the laboratory is in use and by whom. The users can enquire about these conditions in several ways; simple questions can be asked, via voice or text interaction, that require a single data point, e.g. requesting the latest temperature of a specific sensor, "What is the temperature of tsunami?" (a temperature sensor) (See Section V-B for a fuller example). Skills were created with the following intents to allow retrieval of sensor data:
- GetTemperature - queries about air/water temperatures

- GetWaterFlow - questions relating to water flow rate

- GetUser - returns current lab user

The latest temperature readings are also displayed on NodeRED dashboards (see Section $\mathrm{V}-\mathrm{H}$ ) to give an overview of laboratory conditions. More in depth/historical data can also be viewed through a web interface which plots sensor data across a given time period. These interactions allow laboratory users to immediately gain access to pertinent information.

\section{E. Enquire about Laboratory Equipment}

To monitor different laboratory equipment, more sensors were installed and new skills were created enabling users to retrieve vital information about the equipment status. Talk2Lab uses the sensor readings from multiple sources to calculate values such as laser power and wavelength which are then published via MQTT and fed into the Node-RED system. As with the environmental sensors, these values can be retrieved in multiple ways; through dashboards showing the latest sensor readings, or via voice interaction with the following intents:

- GetLaserPower - handles questions about laser power

- GetWavelength - returns laser wavelength

- GetPosition - handles questions on laser beam positions

The GetWavelength intent takes information from a single feed, so does not require slot information. Whereas, the GetLaserPower and GetPosition intents can return the position/power of the Spitfire or Tsunami laser components, so these contain a slot for the equipment. Other sensors were installed to monitor the power consumption of laboratory equipment. However, sampling rates caused the reported values to fluctuate considerably, making the raw data unreliable. Values like these would require real-time processing to provide an average reading, e.g. average of the last 10 readings.

\section{F. Receive Warnings from the Laboratory}

In Talk2Lab, sensors are continually recorded and monitored to trigger warnings and alerts when unexpected conditions are detected (e.g. measurements being outside an acceptable specification). Alert functionality was built into the Node-RED system using Slack interaction, automatically generating Slack alerts for any sensor measurements that were out-of-specification. The alert code was placed into the flows that monitor the sensor feeds so it did not rely on a user making a request to the system, but continuously monitored the readings. If a reading received via MQTT is out-ofspecification a message is generated, and a Slack bot sends the alert to users via a specified channel. An example alert is: "Alert! The temperature of tsunami is out of spec at 28.7 degrees!". This informs the user which piece of equipment is out-of-specification and of its reading, providing the user with information pertaining to the urgency level, and where to fix it. Although the specifications have initially been hard coded, future development could pull specification limits directly from sensor metadata stored in the sensor catalogue. 


\section{G. View Camera Feeds}

Several network enabled cameras were installed in the laboratory which enabled users to visualise outputs from equipment that cannot be directly linked to the network. Computers with outdated operating systems are prohibited from connecting to the university network, and older systems are often not equipped with adequate connectivity. In particular, cameras were installed to capture equipment output used in laser beam alignment, which previously required two people as the output was not visible from where the equipment was adjusted. Integration of the cameras in the Talk2Lab allows alignment by a single person. The camera feeds can be displayed either on a user's laptop or as part of the dashboards displayed on the large screens in the lab, giving the user instant output visualisation whilst adjusting the beam.

Users also often use camera outputs to identify laser behaviour. One output used is the Frequency-resolved Optical Gating (FROG) trace. This output is a spectrogram, used to characterise the laser pulses. Another output is cameras that capture the beam profile at various positions. These are used to determine if the laser beam is released or contained within the shuttered system. These outputs are both captured by network cameras and can be visualised on the large screens or laptops.

\section{H. View Laboratory Dashboards}

Digital Dashboards were implemented to give an overview of multiple information sources within the laboratory. These dashboards were configured using Node-RED and can pull sensor information in using MQTT. Their configuration allows them to be displayed on large display screens within the laboratory and entry area, and remotely on users laptops.

The dashboards are displayed in the laboratory on two display screens (see Fig. 5 \& 6). They are configured to display information in a variety of ways including numbers, plots, gauges, text values and images.

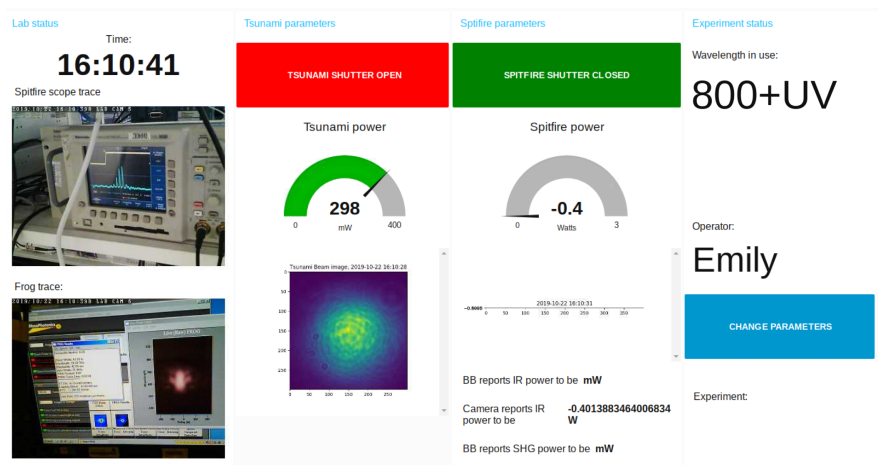

Fig. 5. Entry Room Dashboard: This dashboard is configured to provide an overview of systems before a user enters the main lab room. It pulls information from the cameras and multiple MQTT sensor feeds and colour codes information about laser shutter positions which provides safety information.

This provides significant insight to the user at just a glance and remote access to dashboards gives users an overview of the laboratory from anywhere. Prior to their installation, users entering the laboratory had no knowledge of its state beyond the laser system being on or off. Now users have detailed

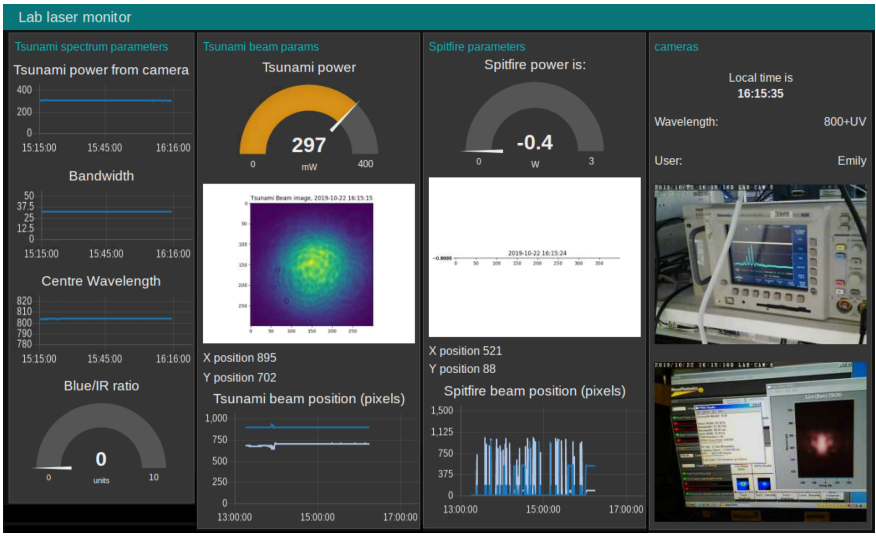

Fig. 6. Main Laboratory Dashboard: This is designed to be seen from a variety of points around the laboratory and provides a more in depth view of the systems. It also pulls in camera feeds, and a wider range of sensors. The plots track a number of measurements, such as beam positions, over time.

information and can make a decision about laboratory safety and equipment use before opening the door.

\section{Helping Users in the Lab}

To improve Talk2Lab's usability, help interactions were created for user guidance. When the system launches, users can receive information on the available intents. A help intent provides request structures for the available intents and sensors so inexperienced users can understand how to use them. Error handling was also implemented, as requests may not always be processed as expected. If requests are misunderstood by Alexa's processing, or if no intent is identified then it returns a generic error; however, in the Node-RED system, error messages were designed to be informative rather than just returning 'error'. In the GetTemperature intent the sensor slot is required; if no value is identified then it returns "Sorry I did not detect a sensor name". If the value identified is not a sensor, it returns "slotvalue is not a valid sensor". The GetLaserPower intent has no slots but will check if the power monitor is active, if not it returns, "The beaglebone is unavailable, please reboot."

\section{TAlk2Lab Evaluation}

The Talk2Lab laboratory currently has ten users who all run experiments, sometimes concurrently. Given the small size we ran group interviews to evaluate Talk2Lab. This evaluation focused on two areas; user evaluation of the implementation of current use cases, and additional functionality that they would like. The Talk2Lab team also evaluated the system from a development and technical perspective.

\section{A. Talk2Lab User Evaluation}

Within user evaluation, laboratory users identified the aspects of the current functionality that they found most useful, and described what needed improving or extending. 
1) Interaction Preferences: The users had different ways of using Talk2Lab depending on their location. Overall, the preferred method was using dashboards to get immediate information. Users noted that whilst they would use text queries or dashboards to check readings outside the laboratory, when inside they tended to check readings directly, or wanted to see a full graphing of the readings. Warning alerts were a popular method for outside the laboratory, so that users could then $\log$ in to the laboratory and directly query the value of a sensor, or access the dashboards for a broader view of what was happening.

2) Voice and Text Interaction: Voice was identified as very useful inside the laboratory, but with requests for additional functionality. Currently voice is used to obtain information, but the users strongest use case was for controlling aspects of the laboratory, with specific requests for control of dashboard content, and laboratory lighting. The Echo Dot functioned well at close range in the laboratory, which had high levels of background noise, understanding most commands up to $1.5 \mathrm{~m}$; however, at further distance commands were often missed. In quieter areas such as the write-up area and entry rooms, this was less of an issue. Further, it was noted that the Echo Dot needed to be louder as sometimes the response could not be heard. Text enquiries were identified as the least useful feature inside the laboratory (as users favoured Alexa or dashboards); however they were noted as useful outside the laboratory to identify issues after alerts. The users found the rigid structure of the text queries quite primitive, and noted that they might use them more if they were more natural.

3) Enquire about Laboratory Environment \& Equipment: Functionality that notified users of experimental issues was identified as the most useful feature. Currently Talk2Lab provides some functionality through monitoring of environment and equipment that gives the users an idea if there is a problem (e.g. if the laser power shows unusual readings, or the temperature in the laboratory is too high). They have identified further features that they would like to be able to monitor:

- Humidity: If the laboratory becomes too humid then the lasers may not operate. (via additional sensors).

- Mode-Lock: If the laser has dropped out of mode-lock then this can be detrimental to the users experiments. (combination of measurements).

- Key Switch Error: If the laser stops, but not due to a switch off procedure or closed shutter, it gives an error that must be rectified. (combination of measurements).

4) Receive Warnings from the Laboratory: Warnings were most useful to users when they alert for potential experiment issues. The users would like to see warnings expanded to include the situations mentioned above (high humidity, loss of mode-lock and key switch errors). They would also like alerts to be customisable for different types of messaging (text, slack, emails), with capacity to assign alerts to different people (e.g. the owner of the experiment being monitored), and to turn them off for one or multiple users. The users also identified desire for Alexa to give severe warnings audibly in the laboratory, and for dashboards to display them.

5) View Laboratory Dashboards: The dashboards have quickly become integral to the laboratory. Users noted that they found them very useful because they display multiple pieces of information at once, and allow users to monitor equipment over time with graphs. Being able to see camera feeds as part of the dashboards was also very helpful. The users wanted extra functionality to control the dashboards via Alexa (e.g. display a single camera feed full screen, or see graphs of multiple sensors side by side). Further, the need for improved mobile interfaces was raised so users can check the laboratory state more easily on phones/tablets.

6) View Camera Feeds: The users appreciate being able to use the camera feeds to set up experiments single-handedly by being able to see and adjust the FROG Trace at the same time. The users noted a need for additional portable cameras that could be moved around the laboratory and pointed at different equipment as necessary. It would be useful for these to run on rechargeable batteries so trailing cables was not an issue.

7) User Desired Functionality:

Controlling the Laboratory: Talk2Lab is currently passive and facilitates information retrieval, but users want to control different aspects of the laboratory such as turning on lasers and fixing errors remotely where possible.

Further Lab Integration: It would be useful to integrate other systems so instructions could be read out from experiment plans, and data/images taken from equipment could be saved automatically in user directories.

Smart Commands: Experiments take different forms, e.g. changes to the laser at specific times. Users would like to tell Alexa about their experiment type and have associated reminders or commands triggered. This would require users to be individually identifiable to Alexa.

Health \& Safety: Talk2Lab mainly monitors for issues relating to experiments, it would be useful to also monitor potential health and safety aspects such as hazardous spillages.

\section{B. Talk2Lab Technical Evaluation}

Talk2Lab was also evaluated from a technical/development perspective, considering current hardware/software limitations.

1) Alexa Limitations: Alexa has several limitations; some which may be overcome by future updates or tweaks to the system, but others may have to be accepted as a compromise for Alexa's other powerful functionalities.

High Background Noise: Unfortunately, it was not possible to interact with Echo Dots through a Bluetooth microphone, which would have solved the problems with background noise.

Structured Language: Alexa requests must follow the strict pattern shown in Fig 4. Although NLP allows some flexibility in requests, users must learn to follow the structure of 'wake word, skill, question', and learn the available intents and parameters, which may require training and time. Help options were incorporated into Talk2Lab to aid training.

Security: Echo Dots have had some security vulnerabilities, previously being hacked and misused to trigger malicious actions or unauthorised purchases [24]. This is currently low concern, as this required physical access and only impacts 2015/16 devices [25]. Currently, Talk2Lab is not very susceptible to misuse; unauthorised users could physically access Talk2Lab, but would be unable to trigger malicious actions 
as the system does not permit controlling equipment or purchasing. However, when laboratory control is added, rigorous security procedures would need to be implemented to ensure unauthorised or accidental actions cannot be triggered.

Privacy: Alexa devices are always listening for the wakeword which activates them. The manufacturers maintain that audio is only streamed following the wake-word, but the always listening aspect may concern users. Whilst not raised in user evaluation, this could be addressed by using the mute feature when Alexa is not required, or push-to-talk devices which only record on a button press. However, these solutions reduce the benefits of hands-free interaction. Furthermore, the Echo Dot integration passes data through Amazon cloud servers, which store history to help the system improve. This data can only be accessed through the Alexa app or web interface with correct login details, but if this is a concern policies may be required to frequently delete this data. If implemented in an environment containing sensitive data the Amazon set-up may not be suitable.

2) Node-RED Limitations: Node-RED was useful for prototyping and outlining the system request flows, but the additional intents and error handling created many nodes, which are difficult to manipulate in the Node-RED visual display. A node.js server may be more manageable for this setup.

3) Overall Limitations: Talk2Lab requires continual internet access as all processing is carried out in the cloud. Within the university installation this is not an issue as the network connection is reliable and is not frequently interrupted. If Talk2Lab was implemented in a network with an intermittent internet connection then a locally hosted system may be necessary. Additionally, in Talk2Lab, many pieces of equipment are controlled by ageing systems which are too specialist and expensive to upgrade regularly to incorporate newer technology. Talk2Lab cannot be easily integrated with this legacy equipment as it cannot be connected to the network.

\section{CONCLUSIONS}

The evaluation showed that Talk2Lab was well received, with certain aspects having become integral to the laboratory users (the group head noted the continual use of dashboards to monitor different laboratory information). The key benefits of Talk2Lab were identified as providing easy access to a wide range of additional data and saving the users time in their work; from using cameras and dashboards to setup experiments quicker, to receiving alerts about potential issues with experiments that need to be addressed. The main suggestions for improvement were to add new features, specifically to control aspects of the laboratory, which will be discussed in Section VIII. The main issues identified with the current implementation are related to the limitations of Alexa, which as noted above may be a compromise for its powerful functionality, or alternative voice systems will need to be considered.

A connected lab environment has many potential uses, both for laboratory users and for collaboration and engagement with a wider audience. Talk2Lab is a starting point to illustrate what can be achieved using sensors and IoT devices; and the current pitfalls of the system (e.g. rigid text interaction through
Slack). Following this, Talk2Lab needs to be developed with further interactions that are both easy to use and have a greater impact on the users daily laboratory work. If laboratories can be remotely accessed or controlled, researchers from different institutions could share resources, or they could be used as educational tools to teach or inspire students and young minds.

Furthermore, new 'smart home assistants' are continuously being released, and incremental developments are also being made to Alexa with new features being released regularly. This means there is continual scope for improvement and developments in this area. Voice interaction could be extended by triggering actions through commands; and linking the recorded sensor/equipment values to an ELN. Moving from information retrieval to triggering events and recording information is a large step towards creating a truly connected lab.

\section{FUTURE WORK}

There are three main areas of Future Work in this project; improving the general functionality of Talk $2 \mathrm{Lab}$, extending the current implementation of Talk2Lab, and expanding its capabilities in other laboratory environments for different scientific disciplines.

\section{A. Improving \& Expanding Functionality}

Voice Interaction: This could be improved by assigning users their own wireless device or headset; enabling the system to recognise and adjust to individual users settings and eliminating high background noise. Alternatively, Alexa voice remotes containing microphones could be used if multiple Echo devices do not cover the laboratory adequately. However, these require investigation to see if they can handle multiple users in the lab at once. Voice interaction could be expanded with complex questions combining sensor measurements to provide further insight, or using dialogues to get additional information. This could be useful for ELN integration, e.g.

1) User: "Save the output of camera1"

2) Alexa: "Where would you like it saved?"

3) User: "Greg's Blog"

Single source questions could also be extended, such as; "How has the temperature of tsunami changed in the last hour?" or "What is the average temperature for tsunami?". However, this requires additional measurements to be stored in Node-RED and necessitates a more robust error handling system, which would also benefit the current set-up.

Slack Text Interaction: This could be made more flexible by using NLP/NLU to allow natural language questions. This would enable requests to be structured similarly to Alexa requests, providing parameters for input into Node-RED flows. This interaction should be directly integrated with the Alexa processing flows to avoid unnecessary replication.

Alerts: Alerts could tag specific users who are listed as being responsible for specific experiments or equipment. These could also be sent via email or text message, and where possible urgent alerts could also be delivered audibly in the lab (via loudspeaker). Functionality could be expanded by giving sensors multiple specification levels (e.g. inner and outer thresholds). Alerts could also be tweaked so they do 
not continuously repeat if a reading is still out of specification (e.g. temperatures report each minute, and cause frustration if sent each minute until fixed). Instead, only repeating if the reading has gone back into specification, or if it is still outof-specification after a specified time period. Although high impact alerts should be notified frequently until fixed.

Adding Cameras \& Image Analysis: As per the user evaluation, portable cameras could be added allowing users to monitor different areas as required. Extra functionality could be applied though additional image analysis of camera outputs, such as the non-networked FROG trace. Currently users examine the visual output displayed in the lab and manually copy the output to another machine if further analysis is required. Image analysis would allow laser behaviour to be accessed directly through Talk2Lab; either by applying it to screen capture from the isolated PC to identify the image shape and corresponding pulse behaviour; or using it within experiments to measure roundness from images. Using image analysis on experimental output could be the first step towards automating the experimental run.

\section{B. Future Vision of Talk2Lab}

Our vision for Talk2Lab exploits the full connection of the physical and digital laboratories across different scientific domains, utilising a range of digital entertainment technologies as shown in Figure 7. Laboratories could identify their occupants, adjust to users preferred settings and display relevant information at their workstations. Instruments could be connected with measurements seamlessly recorded into ELNs eliminating the need for paper lab notebooks. Voice interactions allow users to talk to instruments, enquire about different systems, request readings and even set experiments running. The laboratory environment could be monitored continuously for incidents and irregular readings, triggering both audible alerts and emails to relevant users, and experiments could be monitored and adjusted remotely. This could be the reality of the lab of the future, and Talk2Lab brings us closer to this vision.

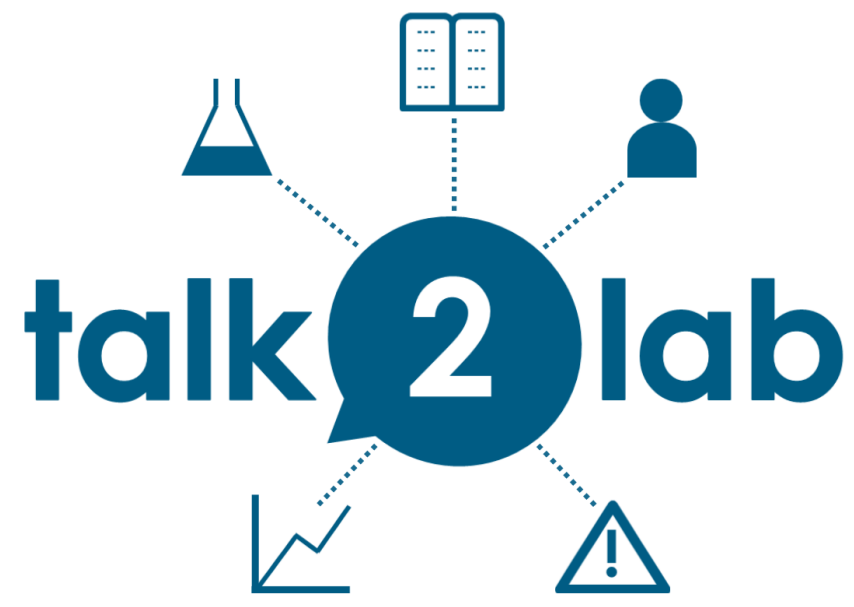

Fig. 7. Future Vision of Talk2Lab

- ELNs / LIMS: Linking with ELNs/LIMS to assist during experiments, such as asking Talk2Lab how much of this chemical do I need to weigh out? Talk2Lab could also link with Inventory Software to automatically update when chemicals are used and notify if stocks are low.

- User Interaction: With this expanded functionality, Talk2Lab could function as a Smart Assistant, enabling users to access and control all aspects of the laboratory.

- Safety: Talk2Lab could provide health \& safety information about experiments e.g. this must be carried out in a fume hood or wear additional protective gloves.

- Data: Talk2Lab could capture data from multiple sources and record it in an ELN (e.g. data from equipment or snapshots from networked cameras).

- Equipment: Talk2Lab could control different pieces of equipment in the laboratory, e.g. users could turn on or shut down lasers remotely. Equipment failure could be predicted by combining multiple sensor readings, e.g. the combination of a temperature increase and a change in the laser beam position could indicate laser failure.

\section{ACKNOWLEDGEMENTS}

This work was supported by Digital Economy IT as a Utility Network under Grant No. EP/K003569/1, Physical and Digital Science South EP/P511407/1, and inherited much from the eScience grants EP/C008863/1 and EP/F05811X/1. SK would like to thank EPSRC for studentship for the Web Science Centre for Doctoral Training at the University of Southampton, funded by the UK Engineering and Physical Sciences Research Council (EPSRC) under Grant No. EP/G036926/1. The authors would like to thank Andy Stanford-Clark (IBM) for his invaluable advice and support in conducting this work. We also thank Ian Stephenson (Bournemouth University) for advice on the use of digital entertainment technologies, and Colin Bird for help in preparation of the manuscript.

\section{REFERENCES}

[1] Elemental, "What do the IoT and R\&D Labs Have in Common? Not Much. Yet," [Online]. Available: https://elementalmachines.io/ iot-rd-labs/, 2017, [Accessed 31-Jan-2020].

[2] J. G. Frey, "Dark Lab or Smart Lab: The challenges for 21st century laboratory software," Organic Process Research \& Development, vol. 8, no. 6, pp. 1024-1035, 2004.

[3] J. M. Robinson, J. G. Frey, A. J. Stanford-Clark, A. D. Reynolds, and B. V. Bedi, "Sensor Networks and Grid Middleware for Laboratory monitoring," in e-Science and Grid Computing, 2005. First International Conference on. IEEE, 2005, pp. 8-pp.

[4] S. Wilson and J. Frey, "The smartlab: Experimental and environmental control and monitoring of the chemistry laboratory," in Collaborative Technologies and Systems, 2009. CTS'09. International Symposium on. IEEE, 2009, pp. 85-90.

[5] D. Locke, "Mq telemetry transport (mqtt) v3. 1 protocol specification," IBM developerWorks Technical Library, vol. 15, 2010.

[6] J. Giles, "The digital lab: lab-management software and electronic notebooks are here-and this time, it's more than just talk." Nature, vol. 481, no. 7382, pp. 430-431, 2012.

[7] G. Hughes, H. Mills, D. De Roure, J. G. Frey, L. Moreau, M. C. Schraefel, G. Smith, and E. Zaluska, "The Semantic Smart Laboratory: a System for Supporting the Chemical eScientist," Organic \& Biomolecular Chemistry, vol. 2, no. 22, p. 3284, 2004.

[8] D. S. Lütjohann, N. Jung, and S. Bräse, "Open source life science automation: design of experiments and data acquisition via "dial-adevice"," Chemometrics and Intelligent Laboratory Systems, vol. 144, pp. 100-107, 2015.

[9] Scientific Computing World, "Building a Smart Laboratory 2018," [Online]. Available: https://www.scientific-computing.com/sites/default/ files/content/BASL18 \\%20Web.pdf, 2018, [Accessed 31-Jan-2020]. 
[10] J. Shumate, P. Baillargeon, T. P. Spicer, and L. Scampavia, "Iot for realtime measurement of high-throughput liquid dispensing in laboratory environments," SLAS TECHNOLOGY: Translating Life Sciences Innovation, vol. 23, no. 5, pp. 440-447, 2018.

[11] Q. Linlin, L. Linjian, S. Chun, W. Gang, and W. Yunlong, "Implementation of iot-based greenhouse intelligent monitoring system," Transactions of the Chinese Society for Agricultural Machinery, vol. 46, no. 3, pp. 261-267, 2015.

[12] R. Roe, "Hacking Science," Scientific Computing World, vol. August/September 2018, no. 161, pp. 16-17, 2018, [Online]. Available: https://content.yudu.com/web/tzly/0A43coj/SCWaug18/html/ index.html?origin=reader [Accessed 31-Jan-2020].

[13] S. Kanza, C. Willoughby, N. Gibbins, R. Whitby, J. G. Frey, J. Erjavec, K. Zupančič, M. Hren, and K. Kovač, "Electronic Lab Notebooks: Can they replace Paper?" Journal of Cheminformatics, vol. 9, no. 1, p. 31, 2017.

[14] S. Kanza, N. Gibbins, and J. G. Frey, "Too many tags spoil the metadata: investigating the knowledge management of scientific research with semantic web technologies," Journal of cheminformatics, vol. 11, no. 1, p. 23, 2019.

[15] B. Halford, "Meet your new Lab Assistant," Chem. Eng. News, vol. 95 , no. 19 , pp. 26-27, 2017, [Online] Available: https://cen.acs.org/articles/ 95/i19/Meet-your-new-lab-assistant.html [Accessed 31-Jan-2020].

[16] Helix, "Helix," [Online]. Available: http://www.askhelix.io/, 2018, [Accessed 31-Jan-2020].

[17] Rick Mullin, "Alexa and your phone are getting schooled in chemistry," Chem. Eng. News, vol. 97, no. 36, 2019, [Online]. Available: https://cen.acs.org/business/informatics/ Alexa-phone-getting-schooled-chemistry/97/i36 [Accessed 31-Jan2020].

[18] J. M. Perkel, "The Internet of Things comes to the Lab," Nature, vol. 542, no. 7639, pp. 125-126, 2017.

[19] M. Poongothai, P. M. Subramanian, and A. Rajeswari, "Design and implementation of iot based smart laboratory," in 2018 5th International Conference on Industrial Engineering and Applications (ICIEA), 2018, pp. 169-173.

[20] J. Austerjost, M. Porr, N. Riedel, D. Geier, T. Becker, T. Scheper, D. Marquard, P. Lindner, and S. Beutel, "Introducing a virtual assistant to the lab: A voice user interface for the intuitive control of laboratory instruments," SLAS TECHNOLOGY: Translating Life Sciences Innovation, vol. 23, no. 5, pp. 476-482, 2018. [Online]. Available: https://doi.org/10.1177/2472630318788040

[21] P. Fox and D. McGuinness, "TWC Semantic Web Methodology," Tetherless World Constellation, 2008.

[22] F. Santiago, P. Singh, L. Sri et al., Building Cognitive Applications with IBM Watson Services: Volume 6 Speech to Text and Text to Speech. IBM Redbooks, 2017.

[23] M. Bostock, V. Ogievetsky, and J. Heer, " $\mathrm{D}^{3}$ data-driven documents," IEEE transactions on visualization and computer graphics, vol. 17, no. 12, pp. 2301-2309, 2011.

[24] C. Wueest, "A Guide to the Security of Voice-Activated Smart Speakers," White Paper. Available: https://www.symantec. $\mathrm{com} /$ content/dam/symantec/docs/security-center/white-papers/ istr-security-voice-activated-smart-speakers-en.pdf, 2017, [Accessed 31-Jan-2020].

[25] M. Barnes, "Alexa, Are you Listening," [Online]. Available: https://labs. mwrinfosecurity.com/blog/alexa-are-you-listening, 2017, [Accessed 31Jan-2020]. 